\section{Comment on Helicobacter pylori seroprevalence and the occurrence and severity of psoriasis - Reply*}

\author{
Priscila Miranda Diogo Mesquita ${ }^{1}$ \\ Augusto Diogo Filho ${ }^{2}$ \\ Miguel Tanus Jorge ${ }^{3}$ \\ Alceu Luiz Camargo Villela Berbert ${ }^{4}$ \\ Sônia Antunes de Oliveira Mantese ${ }^{4}$ \\ José Joaquim Rodrigues ${ }^{4}$ \\ DOI: http:/ /dx.doi.org/10.1590/abd1806-4841.20177256
}

Dear editor,

As mentioned in the article in question, the selected control group had 21 volunteers without skin diseases and without gastrointestinal symptoms. ${ }^{1}$ This group was composed of people with similar socioeconomic status as the patients and who were accompanying those patients during the visits or who had been attended at a neighboring outpatient clinic. We agree that our control group had a small number of participants because of the difficulty in getting healthy people willing to undergo blood collection without any material gain in return.

The methods for the diagnosis of Helicobacter pylori are divided into invasive and non-invasive. In our study, we used the Elisa serological test, a noninvasive method ideal for epidemiological studies, based on the identification of H. pylori-specific IgG antibodies in the patient's serum. However, the has some restrictions. It only detects host exposure to the bacterium, without diagnosing active infection (true infection) (Krogfelt et al., 2005). ${ }^{2}$ We chose this method due to its reduced cost and the low complexity of accomplishment in relation to other diagnostic methods. We believe that the patient's contact with $H$. pylori alone is sufficient to trigger the immunological cascade implicated in the pathogenesis of psoriasis. As in our study, Qayoom and Ahmad detected H. pylori antibodies in $40 \%$ of psoriasis patients and $10 \%$ of control subjects (healthy subjects without gastrointestinal complaints) and concluded that $H$. pylori plays a causal role in the pathogenesis of psoriasis. ${ }^{3}$ Similarly, Fathy et al. compared 20 plaque psoriasis patients with 20 healthy volunteers, matched for age

\footnotetext{
Received on 30.04.2017

Approved by the Advisory Board and accepted for publication on 04.07.2017

* Work performed at Dermatology outpatient service at Universidade Federal de

Uberlândia (UFU) - Uberlândia (MG), Brazil.

Financial support: None.

Conflict of interest: None.

1 Dermatologist - Uberlândia (MG), Brazil

2 Department of Surgery at Universidade Federal de Uberlândia (UFU) - Uberlândia (MG), Brazil.

3 Department of Internal Medicine at Universidade Federal de Uberlândia (UFU) Uberlândia (MG), Brazil.

4 Department of Dermatology at Universidade Federal de Uberlândia (UFU) Uberlândia (MG), Brasil.

C2017 by Anais Brasileiros de Dermatologia
}

and gender, and tested them for H. pilory antibodies using the Elisa test. The mean prevalence of seropositivity in psoriatic patients was significantly higher when compared to controls. Also, the high values correlated with the severity of the disease. They concluded that there is a link between $H$. pylori and psoriasis and that the bacterium may also influence the pathogenesis of the disease. ${ }^{4} \square$

\section{REFERENCES}

1. Mesquita PM, Diogo-Filho A, Jorge MT, Berbert ALCV, Mantese SAO, Rodrigues JJ. Relação da soroprevalência do Helicobacter pylori com a psoríase e sua gravidade. An Bras Dermatol. 2017;92:52-7.

2. Krogfelt KA, Lehours $P$, Mégraud $F$. Diagnosis of Helicobacter pylori infection. Helicobacter. 2005;10:5-13

3. Qayoom S, Ahmad QM. Psoriasis and Helicobacter pylori. Indian J Dermatol Venereol Leprol. 2003;69:133-4

4. Fathy G, Said M, Abdel-Raheem SM, Sanad, H. Helicobacter Pylori Infection: A possible predisposing factor in chronic plaque-type psoriasis. J Egypt Women Dermatol Soc. 2010;7:39-43.

\section{MAILING ADDRESS: \\ Priscila Miranda Diogo Mesquita \\ Av. Pará, 1720 - Bairro Umuarama \\ 38405-320 Uberlândia, MG, Brazil \\ E-mail:primdiogo@yahoo.com.br}

How to cite this article: Mesquita PMD, Diogo-Filho A, Jorge MT, Berbert ALCV, Mantese SAO, Rodrigues JJ. Comment on Helicobacter pylori seroprevalence and the occurrence and severity of psoriasis - Reply. An Bras Dermatol. 2017;92(4):585.

\section{Coexistence of lichen planus follicularis tumidus and Hashimoto's thyroiditis ${ }^{*}$}

Hatice Kaya Ozden ${ }^{1}$

Mualla Polat ${ }^{2}$

Hesna Muzeyyen Astarci ${ }^{3}$

DOI: http://dx.doi.org/10.1590/abd1806-4841.20176708

\section{Dear Editor,}

Lichen planus follicularis tumidus (LPFT) is an extremely rare variant of lichen planus (LP). Clinically, it is characterized by white-to-yellow milia-like cysts and comedones, and red-

\section{Received on 05.06.2016}

Approved by the Advisory Board and accepted for publication on 18.01.2017

* Work performed at the Abant Izzet Baysal University - Bolu, Turquia.

Financial support: None.

Conflict of interests: None.

1 Department of Dermatology - Kocaeli Derince Training and Research Hospital -Kocaeli, Turkey

2 Department of Dermatology - Faculty of Medicine at Abant Izzet Baysal University - Bolu, Turkey

3 Department of Pathology - Faculty of Medicine at Abant Izzet Baysal University Bolu, Turkey

@2017 by Anais Brasileiros de Dermatologia 
dish-to-violaceous plaques. Although it is usually asymptomatic, it may be slightly itchy. ${ }^{1}$ Its most common location is the retroauricular region, but it has also been rarely described in the bilateral retroauricular area and on the cheeks and neck. ${ }^{2-4}$ Histopathology of LPFT shows slightly hyperkeratotic or normal epidermis, which is accompanied by follicles and cysts, filled with orthokeratotic keratin, surrounded by dense lichenoid band-like lymphocytic infiltrate, wedge-shaped hypergranulosis, vacuolar degeneration of the basal zone, pigment incontinence and rare colloid bodies in the papillary dermis. ${ }^{4}$ This characteristic morphology and histopathology of LPFT clearly resembles lichen planopilaris. Here we report a rare case of LPFT concomitant with Hashimoto's thyroiditis.

A 54-year-old female patient presented to our clinic with a pruritic, dark-brown pigmented lesion in the left retroauricular region. The patient noticed the lesion 2 years before but observed no changes in appearance to date. Her medical history was unremarkable. She denied using any topical treatment, cosmetic products, or hearing aids. Physical examination revealed a reticular erythematous hyperpigmented tumid violaceous plaque and 1-2 $\mathrm{mm}$ milia-like white-to-yellow papules (Figure 1). Dermoscopy of the lesion revealed irregular brownish-blue pigment network, milia-like cysts, and comedo-like openings (Figure 1). We observed no hair, nail, or mucous membrane alterations.

Histopathological examination of biopsy specimen taken from the tumid pigmented plaque with milia-like cysts revealed hyperkeratosis, wedge-shaped areas of hypergranulosis, irregular acanthosis, and destruction of the basal layer. The upper dermis showed a band-like lymphohistiocytic infiltrate and prominent pigment incontinence. The papillary dermis revealed some rare areas of eosinophilic colloid bodies (Figure 2). Also, this lymphohistiocytic inflammatory infiltrate surrounded hair follicules and cysts in the dermis. The cysts that were surrounded by stratified squamous epithelium were filled with orthokeratotic keratin (Figure 2). Direct
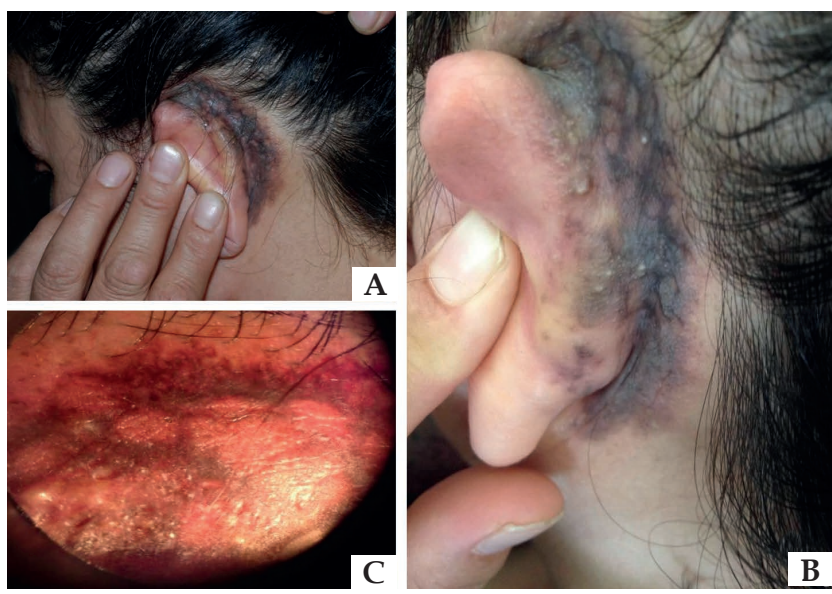

FIGURE 1: A: Tumid reddish/violaceous plaque with white-to-yellow milia-like papules. B: Close-up of the lesion. 1C: Dermoscopic features of the lesion: irregular brownish-blue pigment network, milia-like cysts and comedo-like openings immunofluorescence (DIF) examination was negative. Routine laboratory tests and serological tests were within normal range, but anti-thyroglobulin antibody $(130.8 \mathrm{IU} / \mathrm{ml})$ and anti-thyroid peroxidase antibody $(230.2 \mathrm{IU} / \mathrm{ml}$ ) were very high. The patient consulted with an endocrinologist who performed a thyroid ultrasonography. Her thyroid gland was normal size and nodular formation was not detected. Doppler ultrasonography demonstrated a slight increase in blood flow. She was diagnosed with the subclinical stage of Hashimoto's thyroiditis without any symptoms. Based on the clinical and histological course of the disease, we diagnosed LPFT.

LPFT is characterized by tumid violaceous plaques with multiple white-to-yellow cysts and comedones. It is usually asymptomatic and most commonly affects the postauricular region. Although LPFT has been reported in association with other diseases such as hepatitis B and $C,{ }^{4}$ it has not been reported in association with autoimmune diseases yet.

LPFT can be easily differentiated from other cutaneous diseases. Histopathologically, it can be differentiated from nevus comedonicus by its evolution. Follicular mucinosis is ruled out by the lack of mucin in the follicles. Discoid lupus erythematosus is excluded by DIF and follicular mycosis fungoides with folliculotropism and the presence of perifollicular infiltrate of atypical $\mathrm{T}$ lymphocytes. ${ }^{4}$ However, differentiating LPFT from milia en plaque (MP) and lichen planopilaris may be challenging. Histologically, no lichenoid reaction is found in MP, but it has been speculated that MP is the sequel to previous LPFT. ${ }^{4}$ Lichen planopilaris (also called follicular LP) is a clinical variant of LP and these entities are generally associated with T-cell-mediated autoimmune disorders. ${ }^{5}$ Therefore, patients with LPFT should be thoroughly investigated in terms of autoimmune diseases, as we did with our patient.

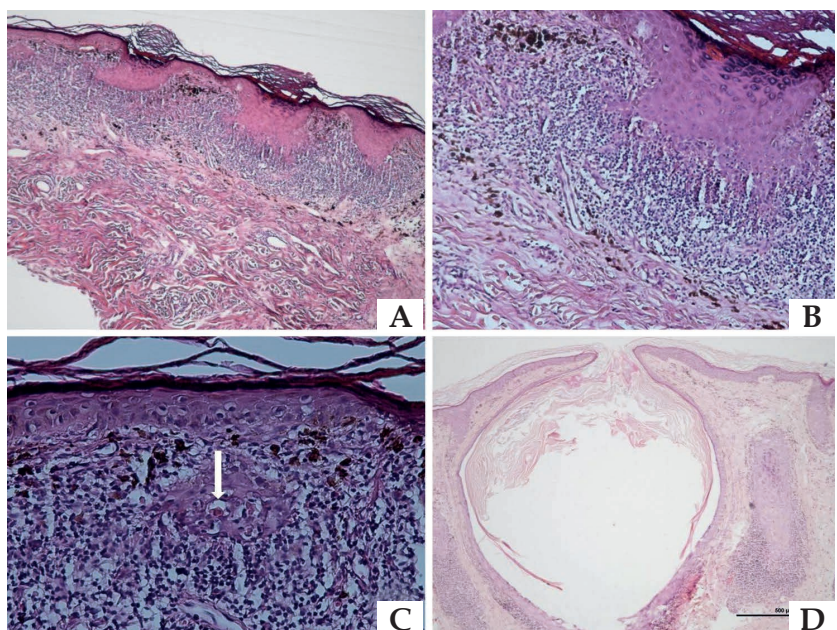

FIGURE 2: A: Hyperkeratosis, wedge-shaped hypergranulosis, irregular acanthosis, and destruction of basal layer (Hematoxylin \& eosin X20). B: The upper dermis had a band-like infiltration of lymphohistiocytes and prominent pigmentary incontinence (Hematoxylin \& eosin X40). C: The papillary dermis reveals colloid bodies (arrow). D: Presence of lichenoid infiltrate surrounding hair follicles and cysts filled with orthokeratin (Hematoxylin \& eosin X20) 
We observed a slightly itchy, tumid, violaceous plaque in our patient only in the left retroauricular region and classic lichen planus lesions or systemic symptoms of hypothyroidism were not present. Histopathological examination, besides the pathognomonic findings of lichen planus, revealed band-like lichenoid infiltrate surrounding the follicles and cysts. Therefore, identifying this entity was critical to reach the LPFT diagnosis and to detect the underlying autoimmune disease.

LPFT tends to be chronic, relapsing, and hard to treat. Consequently, all current treatments available are generally disappointing. In our patient, as LPFT is hard to cure and was localized in a small area, clobetasol propionate cream $0.05 \%$ therapy is recommended despite the retroauricular localization of the lesion. Additionally, we warned our patient to come to our endocrinology department for control as soon as she noticed symptoms of hypothyroidism or every 6-12 months.

We decided to report on this case not only because LPFT is a very rare clinical variant, but also because it was observed for the first time with autoimmune thyroiditis in the present case. $\square$

\section{REFERENCES}

1. Belaïch S, Bonvalet D, Civatte J. Follicular tumidus retro-auricular lichen planus (authors transI). Ann Dermatol Venereol. 1977;104:147-50

2. Grupper Ch, Bensoussan L, Beltzer-Garelly E. Lichen plan folliculaire tumidus rétro-auriculaire bilateral associé a un lichen plan buccal banal leucokératosique et erosif. Ann Dermatol Venereol 1977;104:151.

3. Baptista AP, Rodrigues JB, Cortesão J. Follicular lichen planus tumidus. Ann Dermatol Venereol. 1980;107:1057-9.

4. Jiménez-Gallo D, Albarrán-Planelles C, Linares-Barrios M, Martínez-Rodríguez A, Báez-Perea JM, González-Fernández JA. Facial follicular cysts: a case of lichen planus follicularis tumidus? J Cutan Pathol. 2013;40:818-22.

5. Assouly P, Reygagne P. Lichen planopilaris: update on diagnosis and treatment. Semin Cutan Med Surg. 2009;28:3-10.

MAILING ADDRESS

Hatice Kaya Ozden

Kocaeli Derince Training and Research Hospital

Dermatology Department

41900 Kocaeli / Turkey

E-mail:hatcek@gmail.com

How to cite this article: Ozden HK, Polat M, Astarci MH. Coexistence of lichen planus follicularis tumidus and Hashimoto's thyroiditis. An Bras Dermatol. 2017;92(4):585-7.

\section{Symmetrical drug-related intertriginous and flexural exanthema induced by clarithromycin*}

\author{
Catarina Moreira ${ }^{1,2}$ \\ Ana Paula Cunha ${ }^{1}$
}

\author{
Maria João Cruz ${ }^{1,2}$ \\ Filomena Azevedo
}

DOI: http:/ / dx.doi.org/10.1590/abd1806-4841.20176125

Dear Editor,

A 43-year-old man with no relevant personal background or chronic medication presented with a symmetrical and sharply demarcated erythema on the buttocks and both inner thighs, with a V-shaped pattern, also affecting the lower abdomen and cubital and axillary folds (Figure 1). The patient denied other symptoms or fever. Blood cell count and serum biochemistry analysis were normal. He had been started on clarithromycin for a respiratory infection 7 days before, and denied previous contact with clarithromycin or intake of other medications during the previous weeks. We suspected of cutaneous adverse reaction to clarithromycin, more precisely baboon syndrome, taking into account the pattern of distribution. We suspended the drug and prescribed topical corticosteroids and an antihistamine with significant improvement of the lesions. Two months after complete resolution, we performed patch tests with the Portuguese Contact Dermatitis Group Standard Series and clarithromycin $(1 \%, 5 \%$, and $10 \%$ petrolatum) with a positive result for the drug.

The term "Baboon Syndrome" (BS) was originally introduced to describe a skin eruption resembling the red gluteal area of baboons, noted in 3 patients previously sensitized to mercury, nickel, and ampicillin by topical contact and subsequent systemic exposure to the same substances. ${ }^{1}$

The acronym SDRIFE (Symmetrical Drug-Related Intertriginous and Flexural Exanthema) was proposed for systemically induced cases with the classical baboon distribution pattern and without previous cutaneous sensitization. ${ }^{2}$ In an attempt to better define the terms and classify the cases previously described in the literature, Miyahara et al. divided BS into classical BS (contact allergen-induced, excluding drugs), topical drug-induced BS (contact allergenic drug-induced related to systemic absorption due to topical exposure), systemic drug-induced BS (contact allergenic drug-induced related systemic exposure), and SDRIFE (exposure to

Received on 05.06.2016

Approved by the Advisory Board and accepted for publication on 18.01.2017

* Work performed at the Department of Dermatology and Venereology, Centro Hospitalar de São João EPE - Porto, Portugal.

Financial support: None.

Conflict of interest: None.

${ }^{1}$ Department of Dermatology and Venereology, Centro Hospitalar de São João EPE - Porto, Portugal.

2 Faculty of Medicine, Universidade do Porto - Porto, Portugal.

(C2017 by Anais Brasileiros de Dermatologia 